\title{
The Consortium to Investigate Vascular Impairment of Cognition: Methods and First Findings
}

\author{
Kenneth Rockwood, Heather Davis, Chris MacKnight, Robert Vandorpe, \\ Serge Gauthier, Antonio Guzman, Patrick Montgomery, Sandra Black, \\ David B. Hogan, Andrew Kertesz, Remi Bouchard, Howard Feldman
}

\begin{abstract}
Background: The Consortium to Investigate Vascular Impairment of Cognition (CIVIC) is a Canadian, multi-centre, clinic-based prospective cohort study of patients with Vascular Cognitive Impairment (VCI). We report its organization and the impact of diagnostic criteria on the study of VCI. Methods: Nine memory disability clinics enrolled patients and recorded their usual investigations and care. A case report form included all vascular dementia (VaD) individual criteria for each of four sets (National Institute of Neurological Disorders and Stroke (NINDS-AIREN), Alzheimer's Disease Diagnostic Treatment Centers (ADDTC), the ICD-10 Classification of Mental and Behavioural Disorders (ICD-10), and the Diagnostic and Statistical Manual of Mental Disorders (DSM-IV)) of consensus-based diagnostic criteria and for the Hachinski Ischemia Score (HIS). Investigators, having completed the case report form, were asked to make a clinical judgement about the cognitive diagnosis based on the best available information, including neuroimaging. Results: Of 1,347 patients (mean age 72 years; 56\% women), 846 (63\%) were diagnosed with dementia and 324 (24\%) were diagnosed with VCI. The proportion of patients diagnosed with VaD by the diagnostic criteria was: $23.9 \%$ ( $\mathrm{n}=322$ ) by DSM-IV, $10.2 \%(\mathrm{n}=137)$ by HIS, $4.3 \%(\mathrm{n}=58)$ by ICD-10, 3.8\% $(\mathrm{n}=51)$ by ADTCC, and $3.6 \%(\mathrm{n}=48)$ by NINDS-AIREN. Judged against a clinical diagnosis of $\mathrm{VaD}$, the sensitivity/specificity of each was: DSM-IV (0.77/0.80); HIS (0.41/0.92); ICD-10 (0.29/0.98); ADTCC (0.24/0.98); NINDS-AIREN (0.42/0.995). Compared with a clinical diagnosis of VCI, sensitivities were lower for the diagnostic criteria, reflecting the exclusion of patients who did not have dementia. Conclusions: Consensus-based criteria for VaD omit patients who do not meet dementia criteria that are modeled on Alzheimer's disease. Even for patients who do, the proportion identified with $\mathrm{VaD}$ varies widely. Criteria based on empirical analyses need to be developed and validated.
\end{abstract}

RÉSUMÉ: Le consortium pour l'investigation de la détérioration cognitive vasculaire: méthodes et premières constatations.: Introduction: Le consortium pour l'investigation de la détérioration cognitive vasculaire (CIVIC) est une étude prospective, multicentres, de cohorte de patients suivis en clinique pour une détérioration cognitive vasculaire (DCV). Nous rapportons son organisation et l'impact des critères diagnostiques sur l'étude de la DCV. Méthodes: Neuf cliniques de la mémoire ont recruté des patients et colligé les évaluations et le traitement habituel. Tous les critères individuels de démence vasculaire (DV) de chacun des quatre ensembles de critères diagnostiques identifiés par consensus (NINDS-AIREN, ADTCC, DSM-IV, ICD-10) et du Hachinski Ischemia Score (HIS) ont été notés sur un formulaire d'exposé de cas (FEC). Après avoir complété le FEC, les investigateurs devaient porter un jugement clinique sur le diagnostic cognitif basé sur la meilleure information disponible dont la neuroimagerie. Résultats: 846 des 1347 patients (64\%), dont l'âge moyen était de 72 ans et dont 56\% étaient des femmes, ont reçu un diagnostic de démence et 324 patients (24\%) un diagnostic de DCV. La proportion des patients chez qui on a posé un diagnostic de DV était de 23,9\% $(\mathrm{n}=322)$ selon les critères diagnostiques du DSM-IV; de 10,2\% ( $\mathrm{n}=137)$ selon le HIS; de 4,3\% ( $\mathrm{n}=58$ ) selon le ICD-10; de 3,8\% ( $\mathrm{n}=51)$ selon l'ADTCC et de 3,6\% ( $\mathrm{n}=48)$ selon le NINDSAIREN. En comparant chacun à un diagnostic clinique de démence vasculaire, la sensibilité et la spécificité étaient respectivement de: $0,77 / 0,80$ pour le DSM-IV; 0,41/0,92 pour le HIS; 0,29/0,98 pour l'ICD-10; 0,24/0,98 pour l'ADTCC; 0,42/0,995 pour le NINDS-AIREN. La sensibilité des critères diagnostiques était plus faible que celle du diagnostic clinique de DCV, ce qui reflète l'exclusion des patients qui ne présentent pas de démence. Conclusions: Les critères de DV basés sur les consensus ne tiennent pas comptent des patients qui ne rencontrent pas les critères de démence basés sur le modèle de la maladie d'Alzheimer. Même pour les patients qui rencontrent ces critères, la proportion de ceux chez qui on identifie une DV varie beaucoup. Il faudrait développer et valider des critères basés sur des analyses empiriques.

Can. J. Neurol. Sci. 2003; 30: 237-243

For many years clinicians wishing to formally diagnose vascular dementia $(\mathrm{VaD})$ have faced an array of choices. Several sets of criteria are available, although four dominate. Criteria from the National Institute of Neurological Disorders and Stroke (NINDS-AIREN), ${ }^{1}$ Alzheimer's Disease Diagnostic Treatment Centers (ADDTC), ${ }^{2}$ the ICD-10 Classification of Mental and Behavioural Disorders (ICD-10), 3,4 and the Diagnostic and
From Dalhousie University, Halifax, NS (KR, HD, CM, RV); McGill University, Montréal, PQ (SG); University of Ottawa, Ottawa, ON (AG); University of Manitoba, Winnipeg, MB (PM); University of British Columbia, Vancouver, BC (HF); University of Toronto, Toronto, ON (SB); University of Calgary, Calgary, AB (DBH); University of Western Ontario, London, ON (AK); Université Laval, Quebec, PQ (RB), Canada. ReCeIVED July 12, 2002. ACCEPTED IN FINAL FORM MARCH 13, 2003.

Reprint requests to: Kenneth Rockwood, Queen Elizabeth II Health Sciences Centre, Geriatric Medicine Research Unit, Suite 1421, 5955 Veterans' Memorial Lane, Halifax, Nova Scotia B3H 2E1 Canada. 
Statistical Manual of Mental Disorders (DSM-IV) ${ }^{5}$ each require, to varying degrees, identification of a so-called 'vascular profile' in clinical presentation, and the neuroimaging of vascular lesions. Although the criteria demonstrably are not interchangeable, they share several features. ${ }^{6,7}$ All are similar to the Hachinski Ischemia Score (HIS), ${ }^{8}$ in that they assume a theory of cognitive impairment arising from multiple cerebral infarcts. All require that dementia be present. The criteria for dementia are usually modeled on the dementia of Alzheimer's disease (AD). None is based on evidence - rather each derives from the consensus of experts. As evidence has arisen, it seems not just that the criteria are not interchangeable, but that the extent of misclassification has important consequences for clinical and epidemiological study. ${ }^{9-16}$ In consequence, these studies have given empirical support to the proposal that $\mathrm{VaD}$ should be broadly constituted as vascular cognitive impairment (VCI). ${ }^{17}$

The term VCI refers to a heterogeneous syndrome of cognitive impairment in which a vascular or cardiac cause is implicated. ${ }^{7}$ Three broad clinical subtypes are included: VCI that does not meet dementia criteria (vascular cognitive impairment, no dementia - VascCIND), mixed $\mathrm{AD} / \mathrm{VaD}$ and $\mathrm{VaD}$. These can be further classified radiographically as showing any of cortical or subcortical infarction, or predominantly white matter changes.

Having surveyed the situation in 1993-1994, the Consortium of Canadian Centres for Clinical Cognitive Research (C5R) undertook an empirical study of the manner in which practicing Canadian clinicians diagnose VaD. ${ }^{18}$ Here we report the methods of our inquiry and provide baseline results which estimate the proportion of patients identified as having $\mathrm{VaD}$ by the various sets of criteria, their clinical and neuroimaging profiles, and how these criteria compare with a clinical diagnosis.

\section{METHODS}

Nine memory disability clinics, based in university-affiliated teaching hospitals, participated. Attending physicians were either geriatricians $(n=17)$ or neurologists $(n=14)$. This is a prospective cohort study, with exposures identified at baseline, and outcome measures to include death, institutionalization, and progression of disease. Exposure data included demographic information and data on vascular risk factors. All criteria from each of four sets of diagnostic criteria were recorded in a case report form organized traditionally (i.e., presenting complaint, symptoms, physical exam, neuroimaging results). Items were collected to replicate data collection in the second phase of the Canadian Study of Health and Aging (CSHA). ${ }^{19,20}$ The Consortium to Investigate Vascular Impairment of Cognition (CIVIC) protocol built on usual care at each centre. Although a study nurse completed a semi-structured interview and nurses and physicians were obliged to complete several standardized scales and the diagnostic checklist, use of ancillary investigations, such as neuroimaging (CT and/or MRI, SPECT) and neuropsychological testing, were left to individual sites. The data collection protocol also importantly parallels the clinic-based cohort study known as A Canadian Cohort Study of Cognitive Impairment and Related Dementias (ACCORD), the first report from which has now been submitted for publication.

\section{Diagnostic criteria}

To diagnose VaD, the NINDS-AIREN criteria first define dementia as progressive deficits in memory and other areas of cognition (notably language, motor skills and perception) that occurs in the absence of delirium or "systemic disorders". Similar to NINDS-AIREN, the definition of dementia in DSMIV requires memory and other cognitive deficits. These are specified as being "sufficiently severe to cause impairment in occupational or social functioning" provided that such cognitive and functional impairment represents "a decline from a previously higher level of functioning." The 10th International Classification of Diseases (ICD-10) - Mental and Behavioural Disorders (both clinical and guidelines ${ }^{3}$ and research criteria ${ }^{4}$ ) views dementia as progressive impairment of multiple higher cortical functions, specified as: memory, thinking, orientation, comprehension, calculation, learning capacity, language, and judgement. Note, however, that memory impairment is not specified as primary. ICD-10 also specifies that the deficits occur "in the absence of clouding of consciousness". Of some relevance to the understanding of $\mathrm{VaD}$ is that these criteria note that noncognitive features ("deterioration in emotional control, social behaviour, or motivation") can accompany or even precede dementia. Nevertheless, how this insight should be operationalized is not specified. Like ICD-10, the ADTCC criteria do not privilege memory over other cognitive functions; rather they define dementia as "deterioration from a known or estimated prior level of intellectual function sufficient to interfere broadly with the patient's customary affairs..."2

The criteria also have subtle but important differences in how they view the attribution of vascular injury. The most restrictive criteria (i.e., those most closely on a multi-infarct model) are the ADTCC, which require evidence of two or more strokes outside the cerebellum. The NINDS-AIREN criteria specify the temporal relationship to be within three months of a known stroke, or require that the progression be fluctuating or stepwise. The least restrictive criteria are the DSM-IV, which do not specify the timing of the relationship between ischemic lesions, which allow white matter changes to be sufficient to demonstrate vascular injury, and which permit evidence of focal vascular injury either by neuroimaging or by focal neurological signs by traditional clinical examination.

\section{Other measures}

Although built on a "usual care" protocol, all clinics collected certain standard data, in addition to the case report form data on the elements that went into judgements about dementia and vascular burden of illness. Each of these items was collected in the CSHA. Additional measures were the Mini-Mental State Examination, ${ }^{21}$ the Functional Rating Scale, ${ }^{22}$ the Clinical Dementia Rating, ${ }^{23}$ the Cumulative Illness Rating Scale, ${ }^{24}$ the Disability Assessment for Dementia ${ }^{25}$ and the Functional Assessment Staging Tool ${ }^{26}$ each of which, save the last, was used in the ACCORD study.

\section{Analysis}

The sensitivity and specificity of each set of criteria were calculated in the usual fashion. Yules' Q was calculated to compare the proportions of patients who met each of the various criteria. $^{27}$

\section{Ethics}

All patients gave informed consent. This study was approved by the ethics committee of each participating institution. 
Table 1: Clinical and demographic characteristics of patients by clinical diagnosis of vascular dementia $(\mathrm{VaD})$ and vascular cognitive impairment (VCI)

\begin{tabular}{lccc}
\hline & $\begin{array}{c}\text { Total Sample } \\
(\mathbf{n}=\mathbf{1 , 3 4 7})\end{array}$ & $\begin{array}{c}\text { VaD } \\
(\mathbf{n = 1 0 1})\end{array}$ & \multicolumn{1}{c}{$\begin{array}{c}\text { VCI } \\
(\mathbf{n = 3 2 4})\end{array}$} \\
Age (mean, sd) & $72.4(10.7)$ & $75.4(8.1)$ & $75.6(8.4)$ \\
Female (\%) & $756(56.1)$ & $36(35.6)$ & $147(45.4)$ \\
Years of Education (mean, sd) & $11.3(3.9)$ & $11.2(3.7)$ & $10.9(3.8)$ \\
Hypertension (\%) & $505(37.9)$ & $57(57.0)$ & $179(55.9)$ \\
Lipid Disorders (\%) & $219(17.8)$ & $21(23.6)$ & $67(23.2)$ \\
Diabetes Mellitus (\%) & $168(12.5)$ & $19(18.8)$ & $68(21.3)$ \\
MMSE Score (mean, sd) & $22.3(6.5)$ & $21.4(5.7)$ & $21.9(6.2)$ \\
FRS Score (mean, sd) & $20.0(8.2)$ & $23.4(6.4)$ & $21.6(8.7)$ \\
FAST Score (mean, sd) & $3.5(1.3)$ & $4.2(1.0)$ & $3.8(1.1)$ \\
Focal Neurological & & & \\
$\quad$ Symptoms (\%) & $196(14.6)$ & $50(49.5)$ & $140(43.2)$ \\
Focal Neurological Signs (\%) & $143(10.6)$ & $43(42.6)$ & $85(26.2)$ \\
Number of Focal Neurological & & & \\
$\quad$ Signs (mean, sd) & $1.3(0.7)$ & $1.5(0.7)$ & $1.5(0.7)$ \\
Diagnosed by Neurologist (\%) & $597(44.5)$ & $32(32.0)$ & $122(37.8)$ \\
Diagnosed by Geriatrician (\%) & $745(55.5)$ & $68(68.0)$ & $201(62.2)$ \\
Hachinski Ischemic Score & & & \\
$\quad$ (mean, sd) & $7.7(3.1)$ & $6.2(3.1)$ & $5.1(3.0)$ \\
\hline
\end{tabular}

Table 2: Sensitivity and specificity of each set of diagnostic criteria compared with a clinical diagnosis of vascular dementia $(\mathrm{VaD})$ and vascular cognitive impairment (VCI)

\begin{tabular}{lcccc}
\hline $\begin{array}{l}\text { Diagnostic } \\
\text { Criteria }\end{array}$ & \multicolumn{2}{c}{$\begin{array}{c}\text { Compared with a } \\
\text { dx of VCI } \\
(\mathrm{N}=324)\end{array}$} & $\begin{array}{c}\text { Compared with a } \\
\text { dx of VaD } \\
(\mathrm{N}=101)\end{array}$ \\
\hline NINDS-AIREN & 0.14 & 0.998 & 0.42 & 0.995 \\
ADTCC & 0.14 & 0.99 & 0.24 & 0.98 \\
ICD-10 & 0.13 & 0.99 & 0.29 & 0.98 \\
DSM-IV & 0.53 & 0.85 & 0.77 & 0.80 \\
Hachinski & 0.29 & 0.96 & 0.41 & 0.92 \\
& & & &
\end{tabular}

\section{RESULTS}

The baseline CIVIC cohort was assembled between July 1996 and March 1999. A bi-modal distribution was observed, with most patients being older adults (65+ years), but an important proportion $(9 \%)$ at each clinic being less than age 55 years (median 75; range 37 to 97). As seen in Table 1, vascular risk factors were common, particularly in those diagnosed with VCI of whom $56 \%$ had hypertension, $21 \%$ had diabetes mellitus, and $23 \%$ had lipid disorders, compared with the overall burden of these factors in the study sample $(38 \%, 13 \%$, and $18 \%$ respectively). Most patients (56\%) were women, with the average years of education being $11.3 \pm 3.9$ years. In general, the

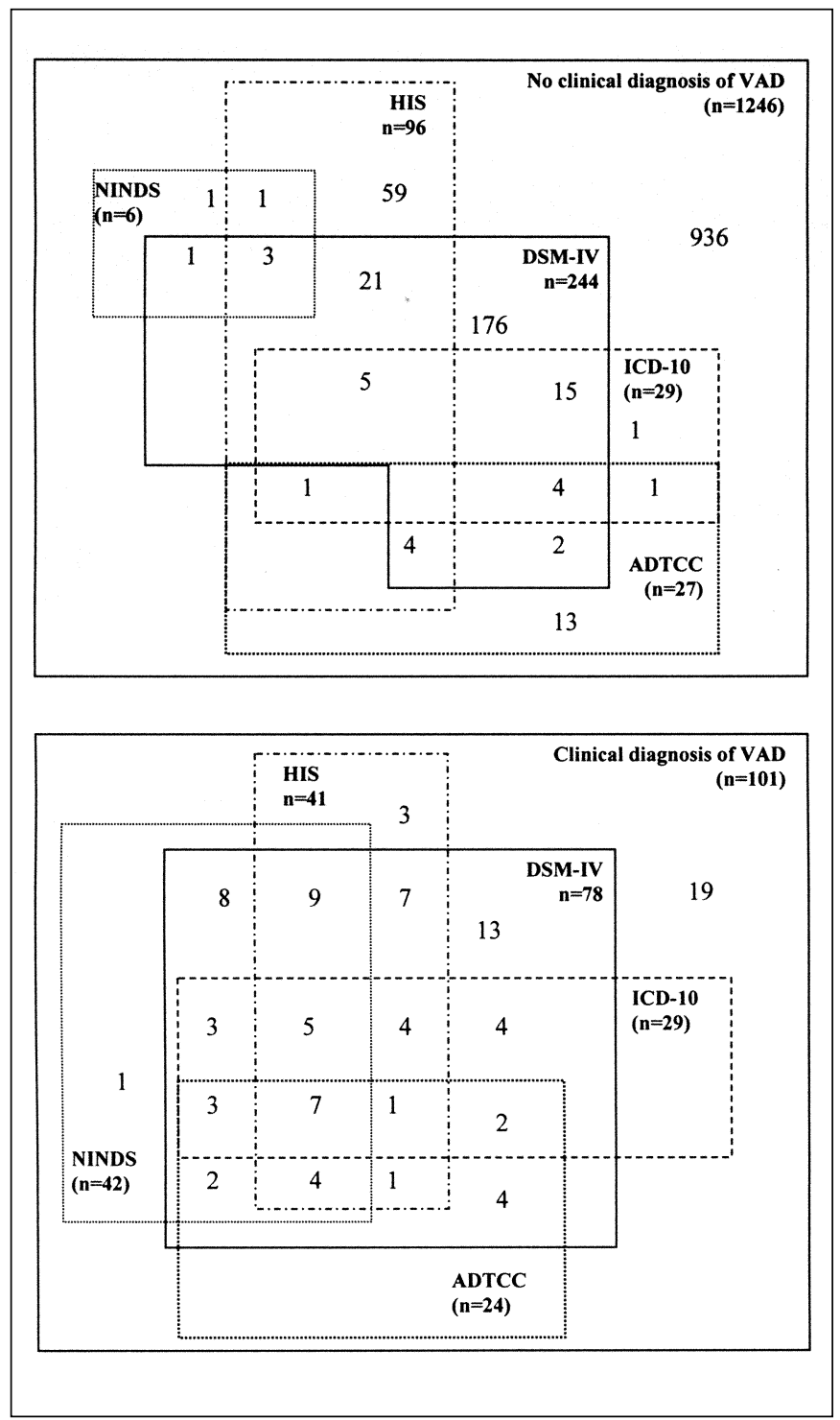

Figure: Subjects identified as having dementia according to various diagnostic classification systems. memory clinics see patients early in the course of their illness, as reflected by a mean MMSE score of $22.3( \pm 6.5)$, as well as a mean functional assessment staging score of $3.6( \pm 1.3)$, where a score of 3 indicates mild cognitive impairment and a score of 4 indicates mild dementia. Although there were many similarities observed between patients seen by geriatricians and those seen by neurologists, patients seen by geriatricians were older $(75 \pm 10$ years compared with $69 \pm 10$ years), and had more comorbid

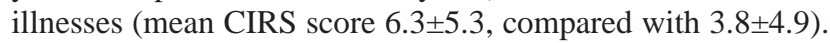

Of the 1,347 patients, the most common diagnosis was probable Alzheimer's disease $(n=463 ; 34 \%)$. Vascular cognitive impairment $(n=324 ; 24 \%)$ was diagnosed in three groups of patients: $97(30 \%)$ who had VCI that did not meet criteria for 
Table 3: Clinical and demographic characteristics of patients identified with vascular dementia by each of four major sets of diagnostic criteria and the Hachinski Ischemia Score (HIS)

\begin{tabular}{|c|c|c|c|c|c|}
\hline & $\begin{array}{l}\text { NINDS-AIREN } \\
(\mathrm{N}=48)\end{array}$ & $\begin{array}{l}\text { ADTCC } \\
(\mathbf{N}=\mathbf{5 1})\end{array}$ & $\begin{array}{l}\text { ICD-10 } \\
(N=58)\end{array}$ & $\begin{array}{l}\text { DSM-IV } \\
(\mathrm{N}=322)\end{array}$ & $\begin{array}{l}\text { HIS } \\
(\mathrm{N}=137)\end{array}$ \\
\hline Age (mean, sd) & $76.2(8.0)$ & $74.8(7.4)$ & $76.8(7.7)$ & $76.0(8.2)$ & $75.3(8.5)$ \\
\hline Female $(\mathrm{n}, \%)$ & $19(40.0)$ & $19(37.3)$ & $28(48.3)$ & $166(51.6)$ & $62(45.3)$ \\
\hline Hypertension (n, \%) & $33(68.8)$ & $32(62.8)$ & $33(57.9)$ & $146(46.4)$ & $91(67.4)$ \\
\hline Lipid Disorders (n, \%) & $8(19.5)$ & $13(30.2)$ & $16(34.0)$ & $47(16.6)$ & $30(25.0)$ \\
\hline Diabetes Mellitus (n, \%) & $12(25.0)$ & $11(22.5)$ & $11(19.3)$ & 47 (14.6) & $37(27.2)$ \\
\hline FRS Score (mean, sd) & $24.2(6.3)$ & $24.3(6.1)$ & $25.1(5.9)$ & $24.2(6.4)$ & $22.5(7.2)$ \\
\hline FAST Score (mean, sd) & $4.4(0.9)$ & $4.3(0.8)$ & $4.4(0.9)$ & $4.3(0.9)$ & $4.0(1.2)$ \\
\hline Focal Neurological Symptoms (n, \%) & $31(64.6)$ & $32(62.8)$ & $37(63.8)$ & $99(30.8)$ & $97(70.8)$ \\
\hline Focal Neurological Signs (n, \%) & $23(47.9)$ & $20(39.2)$ & $29(50.0)$ & $85(26.4)$ & $69(50.4)$ \\
\hline Number of Focal Neurological Signs (mean, sd) & $1.5(0.8)$ & $1.6(0.7)$ & $1.6(0.9)$ & $1.4(0.7)$ & $1.5(0.7)$ \\
\hline Diagnosed by Neurologist (n, \%) & $13(27.1)$ & $26(51.0)$ & $23(39.7)$ & $137(42.8)$ & $32(23.4)$ \\
\hline
\end{tabular}

Table 4: Neuroimaging (CT) characteristics of patients identified by each set of diagnostic criteria

\begin{tabular}{|c|c|c|c|c|c|}
\hline & $\begin{array}{l}\text { NINDS-AIREN } \\
(\mathrm{N}=48)\end{array}$ & $\begin{array}{l}\text { ADTCC } \\
(\mathrm{N}=51)\end{array}$ & $\begin{array}{l}\text { ICD-10 } \\
(\mathrm{N}=58)\end{array}$ & $\begin{array}{l}\text { DSM-IV } \\
(\mathrm{N}=322)\end{array}$ & $\begin{array}{l}\text { HIS } \\
(\mathrm{N}=137)\end{array}$ \\
\hline Single cortical strokes (n, \%) & $9(18.8)$ & $7(13.7)$ & $10(17.2)$ & $41(12.7)$ & $24(17.5)$ \\
\hline Multiple cortical strokes (n, \%) & $4(8.3)$ & $9(17.7)$ & $4(6.9)$ & $14(4.4)$ & $9(6.6)$ \\
\hline Multiple subcortical strokes (n, \%) & $9(19)$ & $33(64.7)$ & $14(24.1)$ & $36(11.2)$ & $13(9.5)$ \\
\hline Clinically significant white matter changes (n, \%) & $23(47.9)$ & $19(37.3)$ & $24(41.4)$ & $156(48.5)$ & $31(22.6)$ \\
\hline Watershed ischemia $(\mathrm{n}, \%)$ & $0(0.0)$ & $0(0.0)$ & $0(0.0)$ & $0(0.0)$ & $0(0.0)$ \\
\hline
\end{tabular}

dementia, 126 (39\%) who were characterized as "possible Alzheimer's disease with a vascular component", and $101(31 \%)$ diagnosed with $\mathrm{VaD}$. The other diagnostic categories were: no cognitive impairment 151 (11\%); cognitive impairment that did not meet the diagnostic criteria for dementia, excluding vascular causes, 253 (19\%); mixed AD with other non-VaD 41 (3\%); frontotemporal dementia 42 (3\%); dementia with Lewy Bodies $32(2 \%)$; other focal dementias $5(0.4 \%)$; and other dementias 37 (3.0\%).

Table 2 represents the sensitivity and specificity of each set of diagnostic criteria compared with a clinical diagnosis of VCI and $\mathrm{VaD}$. Sensitivity for all the diagnostic criteria were relatively low compared with a diagnosis of VCI, and improved slightly when compared with a diagnosis of $\mathrm{VaD}$.

The exact numbers and degree of overlap between the various sets of criteria is illustrated in the Figure, which shows that very few patients would be classified as having $\mathrm{VaD}$ by each set of criteria. As the diagnostic criteria identify different numbers of patients, consideration of the characteristics of patients identified is enabled by exploration of demographic and clinical characteristics of the people identified with VaD (Table 3).

Of the five criteria considered, DSM-IV identified the greatest numbers of patients as having VaD. Lower proportions of these individuals had vascular risk factors and focal neurological signs compared with those identified by other criteria. Table 4 reports the neuroimaging profiles of the patients, using only CT data to allow best comparability. As would be expected, greater proportions of ADTCC classified patients showed a multi-infarct profile, whereas white matter changes were most common amongst those diagnosed by DSM-IV. In general, neuroimaging was felt to change the final diagnosis in $10.8 \%$ of patients.

\section{Discussion}

Eight years ago, the C5R called for empirical studies of the merit of consensus-based criteria for VaD ${ }^{18}$ Since then several studies have shown that the criteria are not substitutable, and also 
lack good inter-rater reliability. ${ }^{9-16}$ This study adds to our understanding of the operational aspects of existing $\mathrm{VaD}$ criteria by profiling other characteristics of those who meet each set. It puts in place the baseline for understanding the criterion validity of each approach as the outcome data become available, and builds on two preliminary reports of mixed dementia ${ }^{28}$ and patterns of presentation ${ }^{29}$ as well as two pilot studies carried out at a single site. ${ }^{30,31}$

We have reported characteristics of the CIVIC cohort at inception. Of the 1,347 patients, 324 had a diagnosis meeting clinicoradiologic criteria for VCI, as operationalized in usual practice at expert referral clinics across the country. Compared with patients with AD, VCI patients were the same age $(75.6 \pm 8.0)$ and had the same years of education $(10.9 \pm 3.8)$, however there was a gender difference with more women in the AD group compared with the VCI group.

Our data must be interpreted with caution. As this is a clinicbased study, and we make no claim that proportions estimated are representative of the population. On the other hand, this study was conducted in parallel with the CSHA, therefore the characteristics of the clinic patients may be understood within that context. For example, $39 \%$ of our patients with cognitive impairment had a diagnosis of $\mathrm{AD}$ vs $37 \%$ of those with a clinical examination in the CSHA. ${ }^{19}$ Similarly, $27 \%$ of our patients with cognitive impairment had a diagnosis of VCI, compared with $24 \%$ of those with a clinical examination in the CSHA. $^{32}$

Some readers might wonder about the usefulness of a clinical diagnosis of $\mathrm{VCI} / \mathrm{VaD}$, and why we did not specify at the outset how physicians should have made such a diagnosis. In our view, to have done so at the outset would have been circular: we would have simply been offering yet another consensus-based set of criteria. We argue that what really is needed are data about how $\mathrm{VaD}$ is diagnosed, given the widespread acknowledgement and empirical studies of the problems with the existing consensusbased criteria. In consequence, the CIVIC protocol built on the usual care provided at each of the participating clinics precisely because no set of diagnostic criteria had been validated. The strategy, therefore, was to use clinical judgement to arrive at a clinical diagnosis and then to compare these judgements with existing criteria. Without such data, there is no non-arbitrary means of specifying at the outset what these criteria should have been. From the present study, we now have some idea of what factors influence the clinical judgment of practicing physicians. Ultimate validation will be available with follow-up, as predictive validity of all criteria, and the clinical judgement can be assayed (e.g., ability to predict death, disease progression) and criterion validity can be compared by reference against a neuropathological diagnosis. In addition, the strategy of building on usual care and following patients to assay outcomes allows the value added by, for example, routine neuroimaging, to be evaluated.

Our data were collected from memory clinics. While inferences drawn in such a setting might be internally valid, their generalizability is not likely to extend to patients seen in cerebrovascular clinics, who more typically present with classical strokes. ${ }^{33}$ At present, however, we do not have a good estimate of what proportions of patients with VCI present to stroke clinics and what proportion come to the attention of memory disorders clinics. In addition, at memory clinics, the pretest probability of $\mathrm{AD}$ is high, so that a memory clinic estimate of VCI includes many patients with so-called "mixed dementia". On the other hand, that too will be the case in representative population samples ${ }^{19}$ including ones with autopsy validation. ${ }^{34-36}$ Thus how "mixed dementia" is handled should not be an afterthought. In practice, and on a population basis, it will be an important aspect of diagnosis. ${ }^{37}$

Our data have some additional important strengths. Coupling this study with the CSHA and ACCORD studies should greatly enhance the efficacy of the inquiry, especially as more detailed analyses of the natural history of various types of VCI are undertaken. Given that the findings are based on usual clinical care, the generalizability, at least to patients with cognitive presentations, is assured. The comparatively low proportion of missing data (apart from neuroimaging, which was undertaken with varying frequencies and modalities across the country, we have no variable with more than $3 \%$ missing data, and most variables have none) speaks to the care with which the study was conducted.

Several recent studies have compared the empirical performance of existing criteria. Briefly, our data are similar to the previous work, in terms of rank order of the number of individuals diagnosed with $\mathrm{VaD}$ by each set of criteria. In a study of 25 cases chosen to represent a spectrum of cognitive impairment and subtypes of dementia, Chui et $\mathrm{al}^{10}$ found that the DSM and modified HIS were most liberal, ADDTC and original HIS were intermediate, and NINDS-AIREN criteria were the most conservative in terms of diagnosing VaD. Wetterling et $\mathrm{al}^{11}$ studied 167 consecutive patients referred for the evaluation of possible dementia, and showed a diagnosis of $\mathrm{VaD}$ for 65 patients $(38.9 \%)$ using the DSM-IV criteria, 28 (16.8\%) using the ICD-10 criteria, $23(13.8 \%)$ using the ADDTC criteria, and $12(7.2 \%)$ using the NINDS-AIREN criteria. They also found that the percentage of overlapping cases between the groups was poor $(<50 \%)$. Verhey et al ${ }^{12}$ studied 124 demented patients from a memory clinic, and found that depending on which criteria were used, frequencies of $\mathrm{VaD}$ ranged from 6 to $32 \%$, and only eight patients were diagnosed as having $\mathrm{VaD}$ by all criteria. In work comparing a neuropathological diagnosis to the clinical criteria, the 'probable' categories of both the ADDTC and NINDS-AIREN criteria in particular lacked sensitivity $(0.21$ and 0.19 respectively). ${ }^{9}$

Interestingly, while the DSM-IV criteria were reasonably sensitive, they lacked specificity, and thus included many people not otherwise classified as having $\mathrm{VaD}$, even by the more liberal criteria that seem to operate in usual practice. It is instructive to consider why this might be so. DSM-IV allows for patients with "dementia due to multiple etiologies" to be double-counted, i.e., included in both $\mathrm{VaD}$ and $\mathrm{AD}$, and $\mathrm{VaD}$ or other dementia groups. This approach accords with a recent proposal to classify mixed dementias as primary neurodegenerative dementia / VCI. ${ }^{16}$ Such an approach can also be operationalized with reasonable precision, using Bayes' theorem, or like modeling approaches. $^{38}$

As noted, the DSM-IV criteria allow for focal neurological symptoms/signs or neuroimaging findings and do not specify a time frame for the strokes to count as being "etiologically related" to the dementia; rather the contribution of ischemic 
injury to cognitive impairment is left to clinical judgment. Such judgments are likely to vary depending on the theory of causality which clinicians bring to bear in relation to the ischemic injuries. As reviewed elsewhere, ${ }^{7,18}$ two poles can be detected: that the cognitive impairment arises directly from the focal impact of infarction, or that the lesions represent a brain at risk for cerebrovascular injury. Whereas the former theory was dominant previously ${ }^{39}$ - when many of these criteria were being decided upon - several lines of evidence (of which, perhaps the most influential are autopsy data showing the ischemic burden of cerebral atherosclerosis on cognition, even with limited frank infarction) point to the latter having substantial credence. ${ }^{34-36}$

The observation of routine clinical practice is of particular value when controversy exists about the nature of clinical phenomena. Under such circumstances, there is merit in going back to basics, and seeing how it is that clinicians operate. Such an exercise, however, can only be a starting point. While it represents an alternative to expert consensus, its real merit is in helping to provide a database from which patterns can be observed. These patterns will be most helpful as they relate to outcome. Put another way, they represent a viable alternative to a "gold standard", which is one form of criterion validity. ${ }^{40}$ Another form of criterion validity (the strongest validation standard) is predictive validity. ${ }^{40}$ With the cohort assembled, we will turn in further studies to understanding whether clinical criteria that can be derived from this study allow us to predict relevant and non-arbitrary outcomes for patients who present with VCI.

\section{ACKNOWLEDGEMENTS}

The CIVIC study was developed through the Consortium of Canadian Centres for Clinical Cognitive Research, supported by the Medical and Research Council of Canada through its MRC/Pharmaceutical Manufacturers' Association of Canada support program in association with Aventis Canada (grant no. PMAC/MRC PA13560 / 1996HMR01). Dr. MacKnight is supported by a New Investigator Award and Dr. Rockwood by an Investigator Award, each from the Canadian Institutes for Health Research. Dr. Rockwood is also supported by the Dalhousie Medical Research Foundation as the Kathryn Allen Weldon Professor of Alzheimer Research.

\section{REFERENCES}

1. Román GC, Tatemichi TK, Erkinjuntti T, et al. Vascular dementia: diagnostic criteria for research studies. Report of the NINDSAIREN International Workshop. Neurology 1993; 43:250-260.

2. Chui HC, Victoroff JI, Margolin D, et al. Criteria for the diagnosis of ischemic vascular dementia proposed by the State of California Alzheimer's Disease Diagnostic and Treatment Centers. Neurology 1992; 42: 473-480.

3. World Health Organization. The ICD-10 Classification of Mental and Behavioural Disorders. Clinical Descriptions and Diagnostic Guidelines. Switzerland: World Health Organization, 1992.

4. World Health Organization. The ICD-10 Classification of Mental and Behavioural Disorders. Diagnostic Criteria for Research. Switzerland: World Health Organization, 1993.

5. American Psychiatric Association. Diagnostic and Statistical Manual of Mental Disorders, 4th ed. Washington DC: American Psychiatric Association, 1994.

6. Rockwood K, Bowler J, Erkinjuntti T, Hachinski V, Wallin A. Subtypes of vascular dementia. Alzheimer Dis Assoc Disord 1999; 13: S59-S65.

7. Erkinjuntti T, Rockwood K. Vascular Cognitive Impairment. Psychogeriatrics 2001; 1: 27-38.
8. Hachinski VC, Iliff LD, Zilhka E, et al. Cerebral blood flow in dementia. Arch Neurol 1975; 32: 632-637.

9. Gold G, Bouras C, Canuto A, et al. Clinicopathological validation study of four sets of clinical criteria for vascular dementia. Am J Psychiatry 2002; 159: 82-87.

10. Chui HC, Mack W, Jackson JE, et al. Clinical criteria for the diagnosis of vascular dementia. A multicenter study of comparability and interrater reliability. Arch Neurol 2000; 57: 191-196.

11. Wetterling T, Kanitz RD, Borgis KJ. Comparison of different diagnostic criteria for vascular dementia (ADDTC, DSM-IV, ICD-10, NINDS-AIREN). Stroke 1996; 27: 30-36.

12. Verhey FR, Lodder J, Rozendaal N, Jolles J. Comparison of seven sets of criteria used for the diagnosis of vascular dementia. Neuroepidemiology 1996; 15: 166-172.

13. Erkinjuntti T, Østbye T, Steenhuis R, Hachinski V. The effect of different diagnostic criteria on the prevalence of dementia. $\mathrm{N}$ Engl J Med 1997; 337: 1667-1674.

14. Lopez OL, Larumbe MR, Becker JT, et al. Reliability of NINDSAIREN clinical criteria for the diagnosis of vascular dementia. Neurology 1994; 44: 1240-1245.

15. Amar K, Wilcock GK, Scott M. The diagnosis of vascular dementia in the light of the new criteria. Age Ageing 1996; 25: 51-55.

16. Ballard C, McKeith I, O'Brien J, et al. Neuropathological substrates of dementia and depression in vascular dementia, with a particular focus on cases with small infarct volumes. Dement Geriatr Cogn Disord 2000; 11: 59-65.

17. Hachinski V. Vascular dementia: a radical redefinition. Dementia 1994; 5(3-4): 130-132.

18. Rockwood K, Parhad I, Hachinski V, et al. Diagnosis of vascular dementia: Consortium of Canadian Centres for Clinical Cognitive Research consensus statement. Can J Neurol Sci 1994; 21: 358-364.

19. The Canadian Study of Health and Aging. Canadian Study of Health and Aging: Study Methods and Prevalence of Dementia. CMAJ 1994; 150: 899-913.

20. The Canadian Study of Health and Aging Working Group. The incidence of dementia in Canada. Neurology 2000; 55: 66-73.

21. Folstein MF, Folstein SE, McHugh PR. "Mini-mental state". A practical method for grading the cognitive state of patients for the clinician. J Psychiatr Res 1975; 12: 189-198.

22. Feldman H, Schulzer M, Wang S, Tuokko H, Beattie BL. The Functional Rating Scale in Alzheimer's disease assessment: a longitudinal study. In: Iqbal K, Mortimer JA, Winblad B, Wisniewski HM, eds. Research Advances in Alzheimer's Disease and Related Disorders. Chichester, England: John Wiley \& Sons, 1995; 235-241.

23. Berg L. Clinical Dementia Rating (CDR). Psychopharmacol Bull 1988; 24: 637-639.

24. Morris JC. The Clinical Dementia Rating (CDR): current version and scoring rules. Neurology 1994; 43: 2412-2414.

25. Gelinas I, Gauthier L, McIntyre M, Gauthier S. Development of a functional measure for persons with Alzheimer's disease: the disability assessment for dementia. Am J Occup Ther 1999; 53: 471-481.

26. Reisberg B. Functional assessment staging (FAST). Psychopharmacol Bull 1988; 24: 653-659.

27. Agresti A. Categorical Data Analysis, John Wiley \& Sons, New York, 1990.

28. Rockwood K, MacKnight C, Wentzel C, et al. The diagnosis of "mixed" dementia in the Consortium for the Investigation of Vascular Impairment of Cognition (CIVIC). Ann N Y Acad Sci 2000; 903: 522-528.

29. Sibley A, MacKnight C, Rockwood K, et al. Living situation and the severity of dementia at diagnosis. Dement Geriatr Cogn Disord 2002; 13: 40-45.

30. Rockwood K, Howard K, MacKnight C, Darvesh S. Spectrum of disease in vascular cognitive impairment. Neuroepidemiology 1999; 18: 248-254.

31. Wentzel C, Darvesh S, MacKnight C, Shea C, Rockwood K. Interrater reliability of the diagnosis of vascular cognitive impairment at a memory clinic. Neuroepidemiology. 2000; 19: 186-193. 
32. Rockwood K, Wentzel C, Hachinski V, et al. Prevalence and outcomes of vascular cognitive impairment. Vascular Cognitive Impairment Investigators of the Canadian Study of Health and Aging. Neurology 2000; 54: 447-451.

33. Bowler JV. Criteria for vascular dementia: replacing dogma with data. Arch Neurol 2000; 57: 170-171.

34. Snowdon DA, Greiner LH, Mortimer JA, et al. Brain infarction and the clinical expression of Alzheimer disease. The Nun Study. JAMA 1997; 277: 813-817.

35. Neuropathology Group of the Medical Research Council Cognitive Function and Ageing Study (MRC-CFAS). Pathological correlates of late-onset dementia in a multicentre, communitybased population in England and Wales. Lancet 2001; 357: 169175.
36. Esiri M, Nagy Z, Smith MZ, Barnetson L, Smith AD. Cerebrovascular disease and threshold for dementia in the early stages of Alzheimer's disease. Lancet 1999; 354: 919-920.

37. Rockwood K. Lessons from mixed dementia. Int Psychogeriatr 1997; 9: 245-250.

38. Mitnitski A, Graham JE, Mogilner AJ, Rockwood K. Vector diagnostics in dementia derived from Bayes' theorem. Am J Epidemiol 1997: 146: 665-671.

39. Tatemichi TK. How acute brain failure becomes chronic: a view of the mechanisms of dementia related to stroke. Neurology 1990; 40: $1652-1659$.

40. Streiner DL, Norman GR. Health Measurement Scales: A practical guide to their development and use. New York: Oxford University Press, 1995. 